\title{
ARTICLE OPEN Graphene wrinkle effects on molecular resonance states
}

\author{
Peter N. Nirmalraj ${ }^{1}$, Kishan Thodkar $\mathbb{D}^{2,3}$, Sarah Guerin ${ }^{4}$, Michel Calame ${ }^{2,3,5}$ and Damien Thompson ${ }^{4}$
}

Wrinkles are a unique class of surface corrugations present over diverse length scales from Kinneyia-type wrinkles in Archean-era sedimentary fossils to nanoscopic crinkling in two-dimensional crystals. Lately, the role of wrinkles on graphene has been subject to debate as devices based on graphene progress towards commercialization. While the topology and electronic structure of graphene wrinkles is known, data on wrinkle geometrical effects on molecular adsorption patterns and resonance states is lacking. Here, we report molecular superstructures and enhancement of free-molecular electronic states of pentacene on graphene wrinkles. A new trend is observed where the pentacene energy gap scales with wrinkle height, as wrinkles taller than $2 \mathrm{~nm}$ significantly screen metal induced hybridization. Combined with density functional theory calculations, the impact of wrinkles in tuning molecular growth modes and electronic structure is clarified at room-temperature. These results suggest the need to rethink wrinkle engineering in modular devices based on graphene and related 2D materials interfacing with electronically active molecules.

npj 2D Materials and Applications (2018)2:8; doi:10.1038/s41699-018-0053-7

\section{INTRODUCTION}

Graphene is never flat, even when supported or suspended. Rather, it is a two-dimensional (2D) atomic solid with intriguing topological defects ranging from corrugations such as ripples, wrinkles, ${ }^{2}$ crumples, $^{3}$ and atomic-scale lattice defects ${ }^{4}$ to micrometer scale grain boundaries $s^{5}$ particularly in graphene grown via chemical vapor deposition (CVD). The origin of these defects ${ }^{6,7}$ and their effects on the mechanical ${ }^{8-10}$ and electrical properties ${ }^{11-}$ 13 of graphene are currently being investigated as graphenebased materials progress towards a technological stable state, reflected in the recent surge in patent applications. ${ }^{14}$ In particular, graphene electrodes integrated in nanoscale devices serve as electrical contacts such as top-electrodes in organic solar cells, ${ }^{15}$ bottom-electrodes in flexible electronics ${ }^{16,17}$ and in-plane electrodes $^{18,19}$ in atomic ${ }^{20}$ and single-molecular circuits. ${ }^{21}$ Some commonly used techniques to obtain high-quality graphene include mechanical exfoliation, ${ }^{22}$ epitaxial growth, ${ }^{23}$ atomically clean graphene grown directly on single crystalline silver, ${ }^{24,25}$ CVD growth on untreated copper foils ${ }^{18,19,26}$ and on electropolished $\mathrm{Cu}^{27}$ The exfoliation method involves peeling few-layer graphene films from a piece of graphite. It offers high charge carrier mobility and defect-free graphene, but the small area of the exfoliated flakes and the very limited yield currently precludes the use of exfoliation in large scale applications. It is also possible to grow graphene in layers by sublimation on silicon carbide ( $\mathrm{SiC}$ ) substrates, but the growth conditions make it challenging to control charge carrier density. ${ }^{28}$

By contrast, CVD is a technique that produces large-area highquality graphene films with minimal defects for industrial scale applications, ${ }^{29,30}$ with a wide range of choice of growth substrates and gas precursors. Two further advantages are that the layer thickness of the graphene films fabricated through CVD can be tuned and the graphene can be transferred to a wide range of substrates. $^{31}$ Using the highly flexible and reproducible CVD fabrication technique, we fabricated single-layer graphene films on copper foil using methane as the carbon precursor. The electronic and structural properties of molecular adsorbates on epitaxial graphene have been well characterised ${ }^{23,32-34}$ and the role of graphene edges on molecular conductance gaps ${ }^{35}$ is known. By contrast, the effect of out-of-plane wrinkles (outsized ripples with an aspect ratio larger than 1$)^{36}$ present on CVD-grown graphene (commonly used in the fabrication of graphene electrodes) on the energetic states of molecules remain to be fully clarified. ${ }^{37}$ Further information on site-dependent discrepancies in molecular resonance will aid efforts to predict the performance of large-scale devices integrated with electrodes made from graphene and other 2-D layered materials. ${ }^{38}$

Here we report the adsorption patterns and electronic states of pentacene adsorbed on wrinkled and flat regions of CVD-grown graphene, measured using atomic force microscopy (AFM), scanning tunneling microscopy (STM), and spectroscopy (STS). The topographic information reveals different growth configurations of pentacene which depend on the graphene landscape, from the assembly of ribbon-like architectures on top of the wrinkles to the emergence of island type patterns on flat regions within a single graphene grain. A doubled conductance gap of $(3.0 \pm 0.3) \mathrm{eV}$ was measured for pentacene adsorbed on the wrinkles compared with $(1.3 \pm 0.2) \mathrm{eV}$ for pentacene adsorbed on flat regions. Comparative spectral analysis of pentacene electronic structure as a function of local adsorption profile confirms pronounced electronic decoupling of pentacene adsorbed on wrinkles (height: $1-3 \mathrm{~nm}$ ), which is in stark contrast to pentacene adsorbed on flat-graphene, where the molecular energy levels are broadened by the underlying metal, confirming that flat-graphene is semi-permeable to the tunneling electrons from underlying $\mathrm{Cu}$. Together with atomic-scale modelling and

\footnotetext{
${ }^{1}$ IBM Research - Zurich, Säumerstrasse 4, 8803 Rüschlikon, Switzerland; ${ }^{2}$ Physics Department, University of Basel, Klingelbergstrasse 82, 4056 Basel, Switzerland; ${ }^{3}$ Empa, Swiss

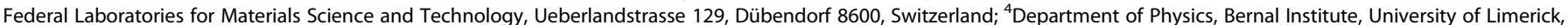
V94T9PX Limerick, Ireland and ${ }^{5}$ Swiss Nanoscience Institute, University of Basel, Klingelbergstrasse 82, 4056 Basel, Switzerland

Correspondence: Peter N. Nirmalraj (peter.nirmalraj@unifr.ch)
}

Received: 6 November 2017 Revised: 25 February 2018 Accepted: 6 March 2018

Published online: 28 March 2018 
density functional theory calculations, our findings provide new insights into molecular adsorption on CVD-grown graphene and highlight the so-far overlooked necessity to consider the influence of topological corrugations on molecular electronic states when designing nanoscale devices with graphene electrodes.

\section{RESULTS AND DISCUSSION}

Figure $1 \mathrm{a}$ is an AFM image of CVD-grown graphene ${ }^{39}$ showing the typical honeycomb structure, seen previously with scanning probe microscopy, ${ }^{5}$ suggesting the conformal growth of graphene following the landscape of the underlying $\mathrm{Cu}$ surface. Spatially magnifying over the AFM image shown in Fig. 1a shows dense wrinkles within the grains as shown in Fig. $1 \mathrm{~b}$ and the corresponding phase image (Fig. 1c). The formation of wrinkles on graphene has been attributed to the non-uniform strain distribution at the physisorbed graphene-copper interface, ${ }^{40}$ differences between the thermal expansion coefficients of graphene and the support metal ${ }^{6}$ during the cooling phase, and also the propagation of defect lines on the metals. ${ }^{41}$ More recent studies identify the pressure exerted by trapped molecules originating from the CVD process ${ }^{40}$ as another possible cause of wrinkle formation. Variations in wrinkle topology $2,36,40$ and related changes in the electronic structure ${ }^{42}$ have been studied in great detail under ultra-high vacuum conditions. We verify the atomicscale geometry of the wrinkles using room temperature STM imaging of CVD-grown graphene samples. Figure 1d is a crosssectional view of a three-dimensionally represented STM image of a typical curved wrinkle on graphene. The height $(\mathrm{H})$ and width (W) of the wrinkle is measured from the high-resolution STM image (Fig. 1d) and the mean $\mathrm{H}$ and $\mathrm{W}$ over 20 such atomically resolved wrinkles is calculated to be $(1.8 \pm 1.0)$ and $(1.1 \pm 0.5) \mathrm{nm}$, respectively. The graphene carbon hexagon is evident from the lateral-view 3-D STM image (Fig. 1e) of the same wrinkle shown in Fig. 1d. The corresponding 2-D unfiltered STM image is shown in Fig. $1 \mathrm{f}$.
Upon registering the variations in CVD-grown graphene topology, we spray-deposited liquid-phase solublized pentacene on graphene. The rationale was to investigate the impact of graphene topology on pentacene assembly, in particular the influence of in-grain wrinkles on the electronic properties of pentacene. Figure 2a shows a large-area AFM topograph of the graphene landscape after pentacene adsorption. A threedimensional pattern is seen with ribbon-like assemblies (marked in yellow) on top of island-type structures (marked in blue) on graphene. Regions with missing molecular layers are also seen within the same AFM image (marked in green), suggesting a discontinuous first molecular layer on graphene. A plausible explanation for the formation of the ribbon-type pentacene structures is that wrinkles on CVD-grown graphene serve as surface templates to guide the pentacene assembly. Spatially magnifying on the ribbon-like structure reveals a two-dimensional configuration as seen from the amplitude (Fig. 2b) and height (Fig. 2c) AFM images which are comparable in geometry to recent reports of pentacene growth modes on $2 \mathrm{D} \mathrm{MoS}_{2}{ }^{43}$ In a more closely related study it was pointed out that the wrinkles on graphene serve as nucleation sites leading to the formation of ribbon-like pentacene ${ }^{44}$ and para-sexiphenyl ${ }^{45}$ molecular structures. In addition to observing pentacene growth differences on wrinkles and flat-graphene, we also measure the local variations in surface roughness parameters of root-mean-square roughness $\left(R_{\mathrm{q}}\right)$, detailed in the schematic in Fig. $2 \mathrm{~d}$. We calculate a mean short-range (area $\left.\leq 100 \mathrm{~nm}^{2}\right) R_{\mathrm{q}}$ of $(2.3 \pm 0.5) \mathrm{nm}$ for pentacene adsorbed on graphene wrinkles (indicated by blue arrow over the AFM image in Fig. 2e) and a mean short-range $R_{\mathrm{q}}$ of $(0.7 \pm 0.2) \mathrm{nm}$ for pentacene adsorbed on flat-graphene (indicated by yellow arrow over the AFM image in Fig. 2e), which is closer to the mean $R_{\mathrm{q}}$ values we calculate for flat-graphene $(0.5 \pm 0.2) \mathrm{nm}$. This variation in the $R_{\mathrm{q}}$ values confirms that the island-type pentacene structures tend to form on the flat-graphene regions between wrinkles, adopting the conformity of closer packed structures with reduced surface roughness compared to pentacene adsorbed on graphene wrinkles. We anticipate that in future experiments it (a)



(d) (b)



(c)

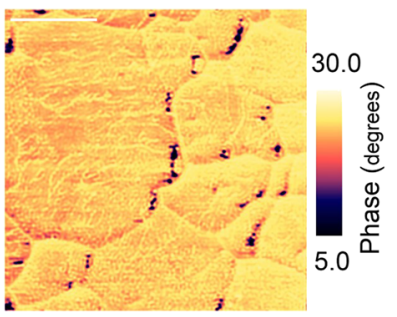

(e)

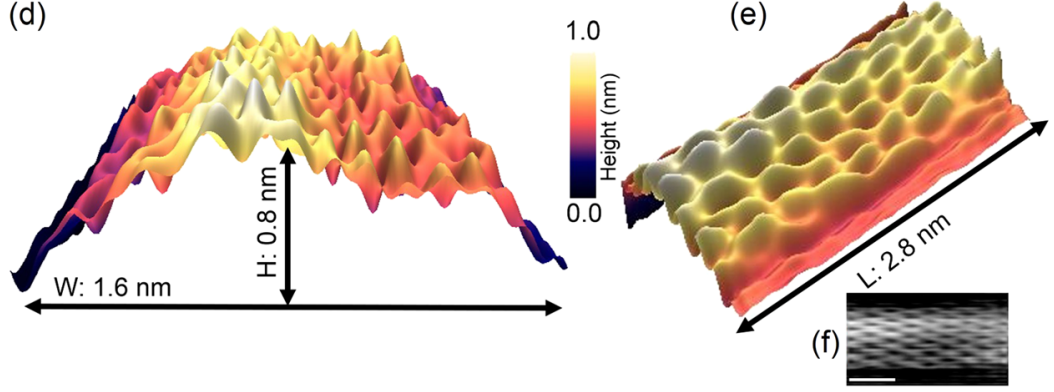

Fig. 1 One-dimensional wrinkle topology on CVD-grown graphene. a Large-area AFM image of CVD grown graphene on copper substrate and $\mathbf{b}$ is a zoom-in of local topography over the region indicated by the white box in panel a and the corresponding phase data $\mathbf{c}$ revealing the wrinkle density (scale bar: $2 \mu \mathrm{m}$ for AFM data in panel a, scale bar: $800 \mathrm{~nm}$ for AFM data in b and c). d Atomic resolution threedimensionally (3D) represented STM image showing the curved nature of the one-dimensional wrinkle geometry (cross-sectional view) of height $(\mathrm{H}: 0.8 \mathrm{~nm})$ and width $(\mathrm{W}: 1.2 \mathrm{~nm})$. e Lateral-view of the 3D constant-current STM image shown in d, highlighting the wrinkle atomic structure with the hexagonally packed carbon framework and the length $(\mathrm{L}: 2.8 \mathrm{~nm})$ along the out-of-plane wrinkle. Tunnelling parameters used for STM data shown (d, e) are $I_{\text {tunnel }}\left(I_{t}\right)=250 \mathrm{pA}, V_{\text {bias }}\left(V_{\mathrm{b}}\right)=1.2 \mathrm{~V}$. f Two-dimensional raw AFM data of the wrinkle shown $(\mathbf{d}, \mathbf{e})(\mathrm{scale}$ bar: $1 \mathrm{~nm})$ 
(a)

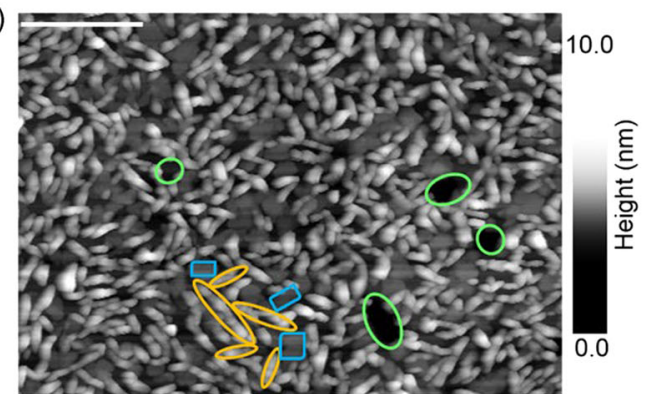

(b)

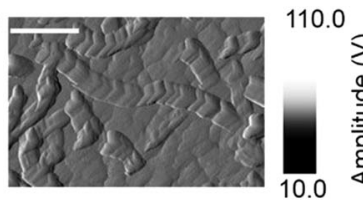

(c)



(d)

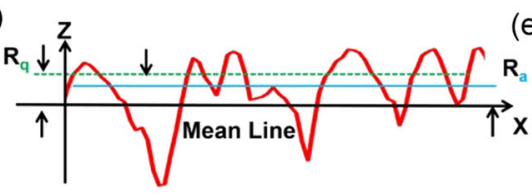

(e)

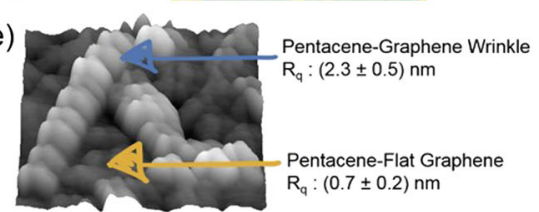

Fig. 2 Pentacene superstructures on wrinkle and flat-area graphene. a Large-area AFM image of pentacene molecular layer spray-deposited onto the CVD graphene surface from liquid-phase. The AFM data shows the formation of chain-like (regions marked in yellow) and island-type (regions marked in blue) pentacene structures within a single graphene grain and areas where pentacene molecular layers are missing (regions marked in green) (scale bar: $1 \mu \mathrm{m}$ ). b, c Spatially magnified AFM amplitude and height data of the chain-like and the island-type pentacene structures. (scale bar: $500 \mathrm{~nm}$ for AFM data (b, c). d Schematic detailing the differences in measurements for root-mean-square $\left(R_{\mathrm{q}}\right)$ and average $\left(R_{\mathrm{a}}\right)$ surface roughness. e Spatially magnified three-dimensionally represented AFM topography over which $R_{\mathrm{q}}$ values are measured for pentacene adsorbed on graphene wrinkle (indicated by blue arrow) and on flat-graphene (indicated by yellow arrow) of ( $2.3 \pm$ $0.5) \mathrm{nm}$ and $(0.7 \pm 0.2) \mathrm{nm}$, respectively. (Scan size for e: $1.1 \times 0.8 \mu \mathrm{m}^{2}$ )

should be possible to reduce the roughness of the pentacene layers when deposited on graphene grown on electropolished $\mathrm{Cu}$, which has lower surface roughness than bare $\mathrm{Cu}$ foil. ${ }^{46}$

The question is then: if pentacene adopts a different growth mode directed by the 1-D wrinkle template, how does the wrinkle height influence molecular resonance states? To address this issue, we performed local point-probe STS measurements on pentacene adsorbed on wrinkles and compared the spectral signature with pentacene adsorbed on flat-region graphene (as shown in the STS schematic in Fig. 3a). Figure $3 \mathrm{~b}$ is a constant-current STM image showing the pentacene coated wrinkles (indicated by red arrow) and flat graphene regions (indicated by yellow arrow). First, the electronic structure of a free-pentacene molecule is determined by density functional theory (DFT) using the Gaussian09 code (Gaussian, Inc) with a B3LYP wavefunction and $6-311++G^{* *}$ basis set. The eigenvalues of the highest occupied molecular orbital (HOMO) and the lowest unoccupied molecular orbital (LUMO) of pentacene are -4.6 and $-2.4 \mathrm{eV}$, respectively, which yields a conductance gap $(\Delta \mathrm{E})$ of $2.2 \mathrm{eV}$ (Fig. 3c). A more rigorous prediction of the gap comes from subtracting lonization Potential and Electron Affinity values estimated from the energies of neutral, radical cation, and radical anion structures, which gives $\Delta \mathrm{E}=4.8 \mathrm{eV}$. The range of values we compute for free (nonbroadened)-pentacene molecule are consistent with literature values $^{47}$ which depend strongly on model chemistry. ${ }^{48,49}$ Figure $3 \mathrm{~d}$ is a differential conductance $(\mathrm{dl} / \mathrm{dV})$ spectrum of pentacene residing along the length of the ribbon structure on top of a wrinkle located by STM as shown in Fig. 3b. Energetically wellresolved molecular resonance peaks are evident from the spectral curve (Fig. 3d) with a distinct region of low conductance for the surface bound pentacene. Two primary non-linear peaks are observed centered around positive $(+1.75 \mathrm{eV})$ and negative bias $(-1.35 \mathrm{eV})$ relative to the Fermi edge, which can be attributed to resonant tunneling through the LUMO and the HOMO levels of surface confined pentacene. Based on spectroscopic analysis at several positions along the pentacene ribbon structure on top of the wrinkle we calculate a mean $\Delta \mathrm{E}$ (energy difference between the HOMO and LUMO-derived resonance peaks) for pentacene adsorbed on the wrinkles of $(3.0 \pm 0.3) \mathrm{eV}$. The differences in the error bars shown in Fig. 4 stems from the variations in the position of the frontier molecular orbital peaks when the spectral curve (forward and reverse) was recorded over ten different locations along the pentacene rows on the graphene wrinkle. In general, the energy gap value we have measured for pentacene on top of a graphene wrinkle at room-temperature is consistent with the recently reported $\Delta \mathrm{E}$ value of $3.3 \mathrm{eV}$ at $5.3 \mathrm{~K}$ for pentacene on $\mathrm{h}-\mathrm{BN}$ atomic surface ${ }^{50}$ but significantly smaller than the $\Delta \mathrm{E}$ value of $4.1 \mathrm{eV}$ at $5 \mathrm{~K}$ for pentacene on highly insulating $\mathrm{NaCl}$ thin film, suggesting that the wrinkles screen but do not completely suppress metal-induced hybridization with the underlying copper substrate. Dispersion corrected $\mathrm{DFT}^{52}$ pentacene-graphene electronic structures (Fig. 3e), computed using methods reported in ref. ${ }^{53}$ show zero electronic interaction between pentacene and graphene; the binding is purely van der Waals, which suggests that pentacene is hybridizing in the experiments with the underlying copper support. The electronic properties of the adsorption complex formed by pentacene on 2D substrates such as BN is known to depend on the geometry of the heterostructures. ${ }^{54}$ In the present calculations we focus on the electronic structure of the complexes between single pentacene molecules and graphene, including also control simulations of pentacene on $\mathrm{Cu}$ (Fig. 5e).

Analyzing the STM/STS dataset, a correlation between the degree of electronic decoupling of the molecules and the height of the wrinkle is observed. Figure 4 is a plot of the measured pentacene molecular energy gap $\Delta E$ as a function of graphene wrinkle height. A trend is visible where $\Delta \mathrm{E}$ scales almost linearly with increase in wrinkle height. A transition phase appears at a wrinkle height of $\sim 2 \mathrm{~nm}$ (shaded in red in Fig. 4) beyond which larger molecular energy gaps are measured and the $\Delta \mathrm{E}$ values tend to plateau (shaded in blue in Fig. 4). This result can be explained from previous STM and STS studies on pentacene electronic structure, where free-molecular electronic properties were reported for pentacene adsorbed on highly insulating $\mathrm{NaCl}$ membranes with a thickness of about three atomic layers, on $\mathrm{Cu}^{51}$ Subsequently, STS based characterization of pentacene adsorbed on flat-graphene regions is performed to understand the role of surface based corrugation of CVD graphene on the energy levels of molecular adsorbates. Figure $5 \mathrm{a}$ shows the $\mathrm{dl} / \mathrm{dV}$ spectra measured after positioning the STM probe within a pentacene island adsorbed on a flat-graphene region. Only weak molecular resonance peaks are detected both at positive and negative 


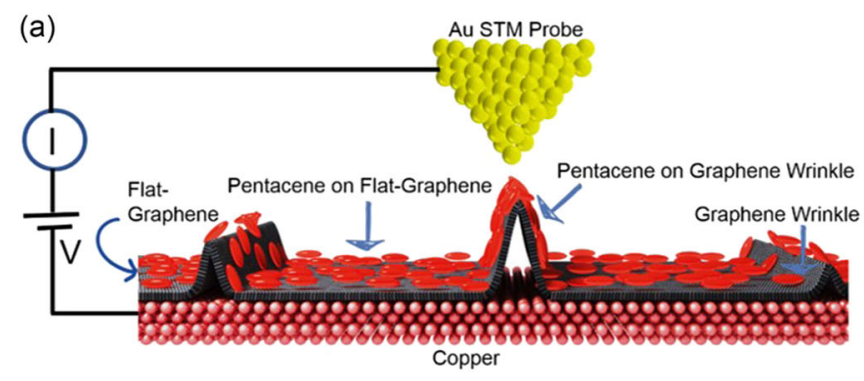

(d)

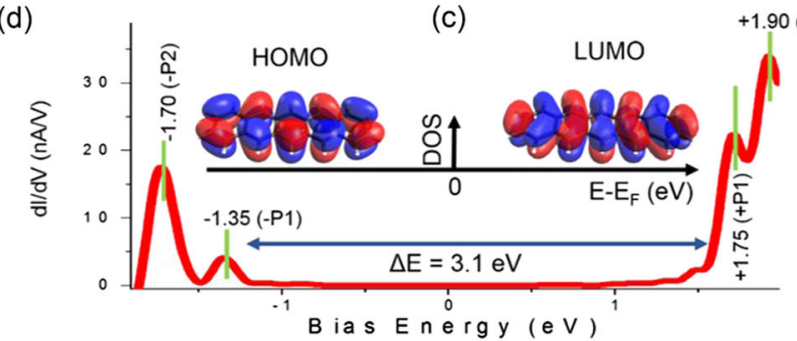

(b)

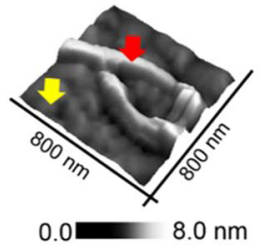

(e)



Fig. 3 Electronically decoupled pentacene on graphene wrinkles. a Schematic detailing the STS setup for local point probe spectroscopy measurements for pentacene adsorbed on CVD-grown graphene landscape on copper (objects shown in schematic not to scale) and the actual constant-current STM map of the pentacene adsorbed on wrinkles (red arrow) and flat-graphene (yellow arrow) is shown (b) (constantcurrent STM recording parameters: $\mathrm{I}_{\mathrm{t}}: 25 \mathrm{pA}, V_{\mathrm{b}}: 100 \mathrm{mV}$ ). c Density functional theory calculated highest occupied molecular orbitals (HOMO) and lowest unoccupied molecular orbitals (LUMO) for gas-phase isolated pentacene molecule. $\mathbf{d}$ Differential conductance (dl/dV) spectrum of pentacene molecules within the row-type structure growth on graphene (as seen from the constant-current STM image (b) revealing sharp frontier molecular resonance peaks. The ribbon-type pentacene structures (red arrow in b) have a propensity to grow directly on the wrinkles on CVD graphene surface which makes them more structurally and electronically decoupled from the underlying copper surface when compared to structures grown on flat graphene (yellow arrow in b). Feedback loop parameters under which the spatially averaged dl/dV spectra were acquired over $\sim 10$ points within the pentacene ribbons: $I_{t}: 150 \mathrm{pA}, V_{\mathrm{b}}: 500 \mathrm{mV}$. e The computed electronic structure of pentacene adsorbed on graphene without underlying copper, shows a purely van der Waals pentacene-graphene binding energy of $-0.9 \mathrm{eV}$ with pentacene positioned at $3.5 \AA$ above the monoatomic graphene surface. Near-HOMO electron density at the top of the valence band (from the Fermi energy level down to $-1 \mathrm{eV}$ below the Fermi level) is overlaid and colored blue in this and all subsequent DFT computed structures with the density plotted at a resolution of 0.025 electrons

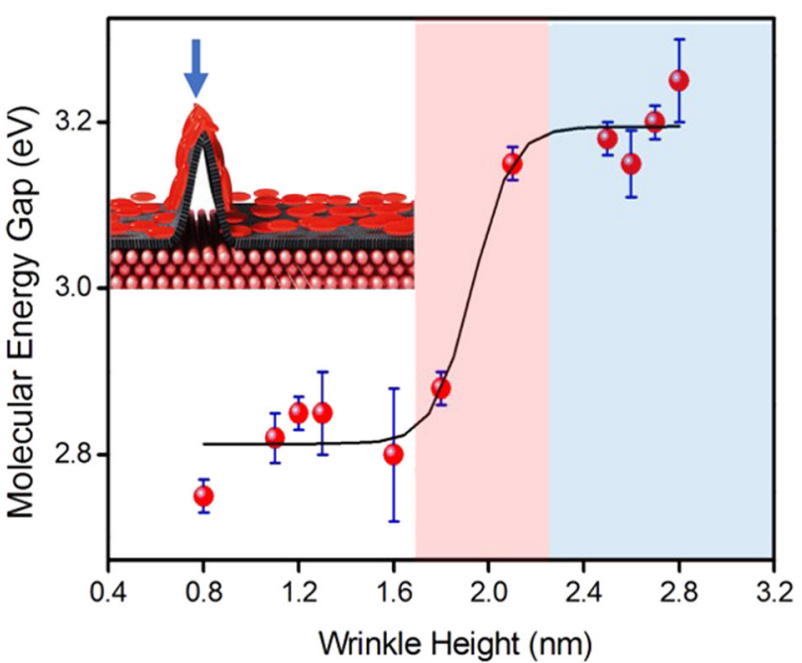

Fig. 4 Effect of wrinkle height on molecular energy gaps. A trend is observed where the measured molecular energy gap $(\Delta \mathrm{E})$ of pentacene scales with the height of the wrinkles on which the molecule is adsorbed (schematically shown in inset, where the blue arrow indicates the position of the STM tip on top of pentacene adsorbed on a wrinkle). A sharp increase is seen in the conductance gap for molecules adsorbed on wrinkles that are taller than $2 \mathrm{~nm}$ (indicated by the red shaded region, with the S-shaped curve overlaid to guide the eye) beyond which the conductance gap is observed to reach its maximum plateau for wrinkles with height up to $3 \mathrm{~nm}$ (indicated by the blue shaded region) voltages, together with a significantly reduced energy gap of $\sim 1.4 \mathrm{eV}$ measured between the two central peaks located at $-0.85 \mathrm{eV}$ and +0.55 . We calculate a mean $\Delta \mathrm{E}$ of $(1.3 \pm 0.2) \mathrm{eV}$ for pentacene adsorbed on flat-graphene based on the statistical analysis shown in Fig. $5 c$, less than half the $\Delta E$ value measured for pentacene adsorbed on 1-3 nm high wrinkles.

The measured weak spectral signature for pentacene on flatgraphene suggests that graphene is semi-permeable to the tunneling electrons from the copper substrate. The inherent hexagonal atomic arrangement together with the presence of known vacancies and topological defects could further increase the probability for a large population of charge carriers to be transmitted through the graphene. As a control, we also measured the electronic coupling of pentacene adsorbed directly on $\mathrm{Cu}$. Figure $5 \mathrm{~d}$ is a dl/dV spectra recorded on pentacene adsorbed on Cu showing a more broadened spectral curve as a result of the direct charge transfer at the pentacene-Cu(111) interface. This is consistent with the electronic interaction calculated between pentacene and bare copper in control DFT simulations (Fig. 5e), confirming that the topology of the spacer layer, in this case graphene, determines the extent to which a molecule can be electronically decoupled from a metallic surface. The differences in the peak widths for the primary molecular resonance peaks for pentacene on graphene-wrinkle, pentacene on flat graphene, and pentacene on $\mathrm{Cu}$ are shown in the comparative full width half maximum (FWHM) analysis (Fig. 5f, g). We attribute the large $\Delta \mathrm{E}$ values and sharper peak widths for pentacene adsorbed on wrinkles to the increased spatial separation created by the wrinkles resulting in significant, systematic screening of $\mathrm{Cu}$ which in turn produces less broadened pentacene resonance states. It should also be taken into account that wrinkles are known to have 


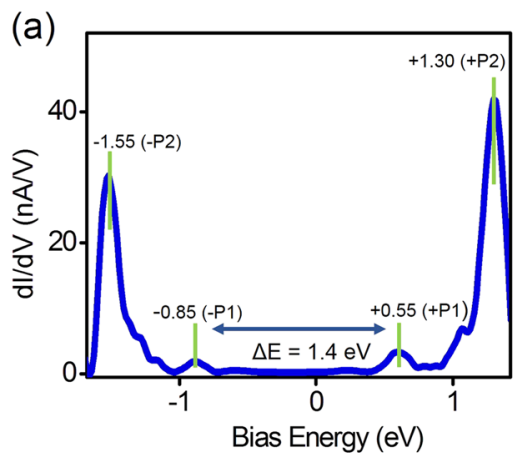

(b)



(c)
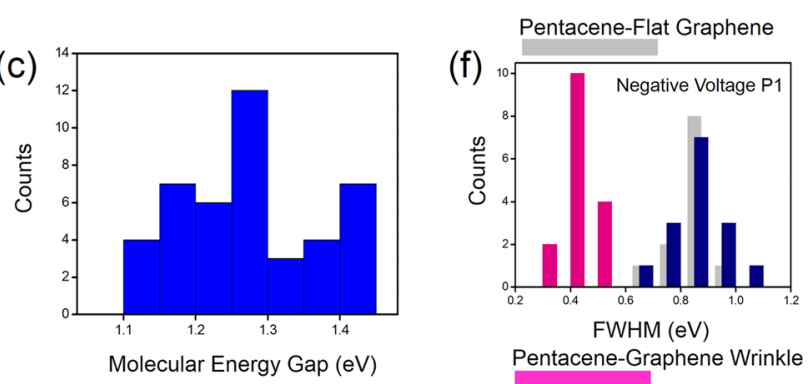

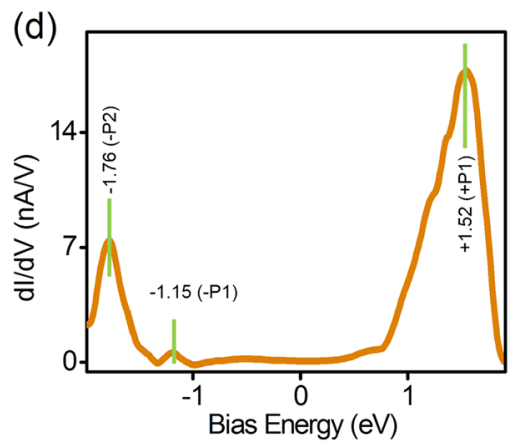

(e)

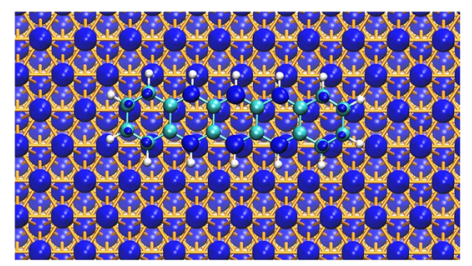

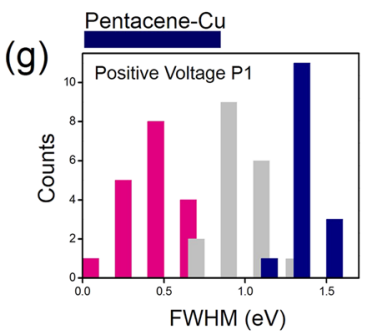

Fig. 5 Metal-induced screening of pentacene resonance states. (a) Spectroscopic signal registered when the STM probe is positioned over pentacene adsorbed on a flat-graphene region (as shown in schematic in b). From the dl/dV spectra the frontier molecular orbital peak positions and a region of low-conductance are discernible. A reduced conductance gap of $1.4 \mathrm{eV}$ is measured between the $\mathrm{HOMO}$ (centered around $-0.85 \mathrm{eV}$ ) and LUMO (centered around $+0.55 \mathrm{eV}$ ). c Statistically binned energy gap values for pentacene adsorbed on the flatgraphene region. d Spectroscopic signal of pentacene adsorbed directly on copper. The dl/dV spectrum shows broadened molecular resonance peaks with weak spectral signature indicating a strong energetic overlap between the adsorbed pentacene and underlying copper, consistent with the known hybridisation of the pentacene LUMO on copper. ${ }^{57}$ e Computed direct pentacene adsorption on copper with a molecular height of $3.0 \AA$ and binding energy of $-3.1 \mathrm{eV}(-1.9 \mathrm{eV}$ electronic interaction due to pentacene-copper hybridisation plus $-1.2 \mathrm{eV}$ van der Waals). f, $\mathbf{g}$ Comparative statistical analysis of full width half maximum peaks (FWHM) for negative voltage peak P1 and positive voltage peak P1 positions for pentacene on graphene wrinkle (pink histogram), pentacene on flat graphene (grey histogram) and pentacene on Cu (blue histogram), respectively. The histograms quantitatively confirm that the primary molecular resonances observed at both positive and negative voltages are significantly sharper for pentacene adsorbed on graphene wrinkles in comparison to pentacene on flat graphene and on $\mathrm{Cu}$

lower electrical conductance than flat graphene as previously reported by $\mathrm{Xu}$ et al. ${ }^{42}$ This limits the contribution to metalinduced effects on molecular resonance states resulting in a larger Coulomb repulsion of the tunneling electrons from $\mathrm{Cu}$. We believe, the preservation of free-molecular electronic structure on wrinkles cannot just be a graphene-specific behavior but can be expected on other related 2-D materials, such as $\mathrm{MoS}_{2},{ }^{55}$ where the wrinkles are spatially separated from the supporting metallic substrates, necessitating future experiments along this direction.

In earlier simulation work the pentacene-copper interface has been examined in terms of band structure, ${ }^{56} \mathrm{DOS}_{1}^{57}$ binding energies, ${ }^{58}$ and changing work functions. ${ }^{59,60}$ For monolayer pentacene, molecules adopt a planar configuration ${ }^{61}$ with explicit mixing of molecular orbitals and metal electronic states. ${ }^{57}$ Toyoda et al. ${ }^{59}$ used vdW-DFT to highlight the strong correlation between work function and the pentacene-copper separation distance. HOMO-LUMO studies have shown that first layer molecular orbitals are strongly affected by metallic d-band orbitals, whereas second layer molecules that are further from the metal surface behave as bulk pentacene. ${ }^{62}$ Bilayer pentacene structures give strong $\mathrm{dl} / \mathrm{dV}$ peaks, whereas LUMO peaks were undetectable for monolayer pentacene. It is possible that the graphene wrinkles begin to simulate bilayer structures by reducing the strength of the metallic d-band interaction. By contrast, band structure DFT calculations show that the electronic states of pentacene and (unsupported) graphene can resonate with each other ${ }^{63}$ (near the $X$ point). The electronic coupling between pentacene and graphene is shown to be four times stronger than that between the pentacene molecules themselves. The graphene-copper interface itself is also well studied-the adsorption of graphene onto copper (and metals generally) causes doping of the graphene sheet, resulting in work function changes and Fermi shifts. $^{64,65}$ Epitaxial growth of pentacene on graphene/copper results in dominant $\pi-\pi$ interactions between the molecules (in a flat configuration) and the surface. ${ }^{66}$ However Lee et al. have shown that molecules begin to adopt an upright configuration for CVD grown pentacene at film thicknesses greater than one molecule, which then become dominated by intermolecular $\pi-\pi$ interactions. ${ }^{67} \mathrm{~A}$ detailed theoretical and experimental study by Zhang et al. of pentacene deposited on atomically flat BN confirms 
the importance of intralayer and interlayer interactions ${ }^{54}$; pentacene molecules that are further from the surface have lower interaction strengths and altered charge transport properties, with negligible influence of the 2D substrate on charge transport beyond a two-molecule pentacene bilayer.

In summary, we demonstrate that atomic scale wrinkles on graphene trigger variations in molecular growth modes and more importantly for device applications, preserve free-molecular electronic states. Our spectroscopy measurements highlight the reality that differently decoupled electronic molecules can exist on metal supported 2-D membranes. The benefit of a wrinkle (which is often projected as a performance limiter) is made clear, and further fuels the debate on whether wrinkles on graphene need be ironed out ${ }^{68}$ or if in fact the wrinkle geometry and density can be modulated in a predictable manner ${ }^{36}$ prior to integration of graphene electrodes in high-density circuits. Our findings indicate that it can, and so wrinkles and similar topological features (both process artefacts and engineered) can potentially serve as platforms in specific cases where the functionality of designer molecules needs to be preserved and later exploited. In the shorter term, the new insights gained on the molecule-wrinkle interfacial effects merits consideration when benchmarking the performance of molecular electronic devices based on nanoelectrodes fabricated from CVD-grown graphene and emerging 2D atomic materials.

\section{METHODS}

Pentacene was purchased in powder form from Sigma Aldrich (99\% purity, CAS number: 135-48-8) and solublized in benzene, and used throughout this study without additional purification. Ten microliter of pentacene solution with a concentration of $10^{-2} \mathrm{M}$ was spray deposited (dual-pass, Harder and Steenbeck, evolution airbrush) onto a CVD-grown graphene sample on copper foils (sample was synthesized using protocols given in ref. ${ }^{53,69}$ ). Immediately following the spray-deposition step the sample was blow dried using $\mathrm{N}_{2}$ gas in a fume cupboard and then fixed on a metal disk (purchased from Ted Pella, Inc) for AFM or placed within a custom-built Teflon based liquid-cell sample holder filled with a thin layer of silicone oil for STM measurements. The silicone oil film protects the organic architecture from ambient contamination ${ }^{70}$ and ensures stable STM imaging $^{71,72}$ and spectroscopy. ${ }^{70,73}$ AFM was conducted on a Dimension $\checkmark$ AFM system operated in tapping mode under standard laboratory conditions with a HI-RES tip ( $\mathrm{Cr}$-Au coating) with tip radius of $<2 \mathrm{~nm}$ purchased from $\mu$ Masch Co. Ltd. STM was performed in constant-current mode at room-temperature using a Veeco Scanning Tunnelling Microscope, Nanoscope Illa, Multimode (Scanner model: E- Scanner) located in a noise-free laboratory. A mechanically cut Au wire $(0.25 \mathrm{~mm}$, Good Fellow $\mathrm{GmbH}$ ) was used as the STM probe. Bias voltage is applied to the sample and the metal tip held at ground potential. The preamplifer has a current sensing capability of $\sim 1 \mathrm{pA}$. For the STS measurements, the Au tip was first positioned on top of either pentacene chain-like structures or on the island-type configuration and then the feedback loop was opened at a fixed height above the molecular region and the voltage is swept from $-2 \mathrm{~V}$ to $+2 \mathrm{~V}$, while the current was being recorded. The feedback loop was reinitiated in-between recording the spectroscopic data to confirm the position of the STM tip is located on top of the specific region of interest. The STS spectra on pentacene presented in this work was recorded using multiple Au tips prepared using identical protocols to check for reproducibility. To avoid tip artefacts the Au tip was calibrated by measuring standard reference differential conductance spectra acquired over blank Au (111) and graphene. ${ }^{70}$ Image processing was performed using Gwyddion 2.39 freeware (http://gwyddion.net).

Data availability

All data related to the manuscript is available on request from the corresponding author.

\section{ACKNOWLEDGEMENTS}

This work was partially supported by the Swiss Federal Institute of Metrology (metas) via the EMRP project GraphOhm (SIB51) and the EC FP7-ITN MOLESCO grant (no.
606728). P.N.N thanks Miguel Spuch Calvar for support with schematics. D.T. acknowledges Science Foundation Ireland (SFI) for financial support under Grant Number 15/CDA/3491, and for provision of computing resources at the SFI/Higher Education Authority Irish Center for High-End Computing (IHEC).

\section{AUTHOR CONTRIBUTIONS}

P.N.N. designed and conducted the liquid-STM, STS and AFM measurements. D.T. and S.G. performed the DFT calculations. K.T. and M.C. provided the CVD grown graphene samples. All authors participated in the analysis and discussion of the data and in writing the manuscript.

\section{ADDITIONAL INFORMATION}

Competing interests: The authors declare no competing interests.

Publisher's note: Springer Nature remains neutral with regard to jurisdictional claims in published maps and institutional affiliations.

\section{REFERENCES}

1. Tapaszto, L. et al. Breakdown of continuum mechanics for nanometrewavelength rippling of graphene. Nat. Phys. 8, 739-742 (2012).

2. Zhu, W. et al. Structure and electronic transport in graphene wrinkles. Nano Lett. 12, 3431-3436 (2012).

3. Zang, J. et al. Multifunctionality and control of the crumpling and unfolding of large-area graphene. Nat. Mater. 12, 321-325 (2013).

4. Banhart, F., Kotakoski, J. \& Krasheninnikov, A. V. Structural defects in graphene. ACS Nano 5, 26-41 (2011).

5. $\mathrm{Yu}, \mathrm{Q}$. et al. Control and characterization of individual grains and grain boundaries in graphene grown by chemical vapour deposition. Nat. Mater. 10, 443-449 (2011).

6. Deng, S. \& Berry, V. Wrinkled, rippled and crumpled graphene: an overview of formation mechanism, electronic properties, and applications. Mater. Today 19, 197-212 (2016).

7. Cerda, E. \& Mahadevan, L. Geometry and physics of wrinkling. Phys. Rev. Lett. 90, 074302 (2003).

8. Nicholl, R. J. T. et al. The effect of intrinsic crumpling on the mechanics of freestanding graphene. Nat Commun. 6, 8789 (2015).

9. Verma, A. \& Parashar, A. The effect of STW defects on the mechanical properties and fracture toughness of pristine and hydrogenated graphene. Phys. Chem. Chem. Phys. 19, 16023-16037 (2017).

10. Kim, K. S. et al. Large-scale pattern growth of graphene films for stretchable transparent electrodes. Nature 457, 706-710 (2009).

11. Bao, W. et al. Controlled ripple texturing of suspended graphene and ultrathin graphite membranes. Nat. Nano 4, 562-566 (2009).

12. Eun-Ah, K. \& Neto, A. H. C. Graphene as an electronic membrane. EPL 84, 57007 (2008).

13. Guinea, F., Katsnelson, M. I. \& Vozmediano, M. A. H. Midgap states and charge inhomogeneities in corrugated graphene. Phys. Rev. B 77, 075422 (2008).

14. Zurutuza, A. \& Marinelli, C. Challenges and opportunities in graphene commercialization. Nat. Nano 9, 730-734 (2014).

15. Liu, Z. et al. The application of highly doped single-layer graphene as the top electrodes of semitransparent organic solar cells. ACS Nano 6, 810-818 (2012).

16. Seo, S., Min, M., Lee, S. M. \& Lee, H. Photo-switchable molecular monolayer anchored between highly transparent and flexible graphene electrodes. Nat. Commun. 4, 1920 (2013).

17. Zhang, Q. et al. Graphene as a promising electrode for low-current attenuation in nonsymmetric molecular junctions. Nano Lett. 16, 6534-6540 (2016).

18. Nef, C. et al. High-yield fabrication of $\mathrm{nm}$-size gaps in monolayer CVD graphene. Nanoscale 6, 7249-7254 (2014).

19. El Abbassi, M. et al. From electroburning to sublimation: substrate and environmental effects in the electrical breakdown process of monolayer graphene. Nanoscale 9, 17312-17317 (2017).

20. Pósa, L. et al. Multiple physical time scales and dead time rule in few-nanometers sized graphene-siox-graphene memristors. Nano Lett. 17, 6783-6789 (2017).

21. Prins, F. et al. Room-temperature gating of molecular junctions using few-layer graphene nanogap electrodes. Nano Lett. 11, 4607-4611 (2011).

22. Martinez, A., Fuse, K. \& Yamashita, S. Mechanical exfoliation of graphene for the passive mode-locking of fiber lasers. Appl. Phys. Lett. 99, 121107 (2011).

23. Cho, J. et al. Structural and electronic decoupling of C60 from epitaxial graphene on SiC. Nano Lett. 12, 3018-3024 (2012).

24. Kiraly, B. et al. Solid-source growth and atomic-scale characterization of graphene on Ag(111). Nat. Commun. 4, 2804 (2013). 
25. Wu, R. et al. Quasi-free-standing graphene nano-islands on $\mathrm{Ag}(110)$, grown from solid carbon source. Appl. Phys. Lett. 110, 213107 (2017).

26. Wan, X., Chen, K. \& Xu, J. Interface engineering for CVD graphene: current status and progress. Small 10, 4443-4454 (2014).

27. $\mathrm{Wu}, \mathrm{X}$. et al. Growth of continuous monolayer graphene with millimeter-sized domains using industrially safe conditions. Sci. Rep. 6, 21152 (2016).

28. Lara-Avila, S. et al. Non-volatile photochemical gating of an epitaxial graphene/ polymer heterostructure. Adv. Mater. 23, 878-882 (2011).

29. Li, X. et al. Large-area synthesis of high-quality and uniform graphene films on copper foils. Science 324, 1312-1314 (2009).

30. Wan, X. et al. High-quality large-area graphene from dehydrogenated polycyclic aromatic hydrocarbons. Chem. Mater. 24, 3906-3915 (2012).

31. Lee, Y. et al. Wafer-scale synthesis and transfer of graphene films. Nano Lett. 10, 490-493 (2010).

32. Wang, Q. H. \& Hersam, M. C. Room-temperature molecular-resolution characterization of self-assembled organic monolayers on epitaxial graphene. Nat. Chem. 1, 206-211 (2009).

33. Jung, M. et al. Atomically resolved orientational ordering of C60 molecules on epitaxial graphene on $\mathrm{Cu}(111)$. Nanoscale 6, 11835-11840 (2014).

34. MacLeod, J. M. \& Rosei, F. Molecular self-assembly on graphene. Small 10, 1038-1049 (2014)

35. Pshenichnyuk, I. A., Coto, P. B., Leitherer, S. \& Thoss, M. Charge transport in pentacene-graphene nanojunctions. J. Phys. Chem. Lett. 4, 809-814 (2013).

36. Pacakova, B. et al. Mastering the wrinkling of self-supported graphene. Sci. Rep. 7, 10003 (2017)

37. Gehring, P. et al. Distinguishing lead and molecule states in graphene-based single-electron transistors. ACS Nano 11, 5325-5331 (2017).

38. Ahn, E. \& Kim, B.-S. Multidimensional thin film hybrid electrodes with MoS2 multilayer for electrocatalytic hydrogen evolution reaction. ACS Appl. Mater. Interfaces 9, 8688-8695 (2017).

39. Nirmalraj, P. N., Lutz, T., Kumar, S., Duesberg, G. S. \& Boland, J. J. Nanoscale mapping of electrical resistivity and connectivity in graphene strips and networks. Nano Lett. 11, 16-22 (2011).

40. Wang, W., Yang, S. \& Wang, A. Observation of the unexpected morphology of graphene wrinkle on copper substrate. Sci. Rep. 7, 8244 (2017).

41. Chae, S. J. et al. Synthesis of large-area graphene layers on poly-nickel substrate by chemical vapor deposition: wrinkle formation. Adv. Mater. 21, 2328-2333 (2009).

42. Xu, K., Cao, P. \& Heath, J. R. Scanning tunneling microscopy characterization of the electrical properties of wrinkles in exfoliated graphene monolayers. Nano Lett. 9, 4446-4451 (2009).

43. Bettis Homan, S. et al. Ultrafast exciton dissociation and long-lived charge separation in a photovoltaic pentacene-MoS2 van der Waals heterojunction. Nano Lett. 17, 164-169 (2017).

44. Chhikara, M., Pavlica, E. \& Bratina, G. Grafold-driven nucleation of pentacene on graphene. Surf. Sci. 609, L5-L8 (2013).

45. Khokhar, F. S. et al. The influence of substrate temperature on growth of parasexiphenyl thin films on Ir $\{111\}$ supported graphene studied by LEEM. Surf. Sci. 606, 475-480 (2012)

46. Zhang, B. et al. Low-temperature chemical vapor deposition growth of graphene from toluene on electropolished copper foils. ACS Nano 6, 2471-2476 (2012).

47. Kaur, l. et al. Substituent effects in pentacenes: gaining control over HOMO-LUMO gaps and photooxidative resistances. J. Am. Chem. Soc. 130, 16274-16286 (2008).

48. Zhang, G. \& Musgrave, C. B. Comparison of DFT methods for molecular orbital eigenvalue calculations. J. Phys. Chem. A 111, 1554-1561 (2007).

49. Faber, C., Boulanger, P., Attaccalite, C., Duchemin, I. \& Blase, X. Excited states properties of organic molecules: from density functional theory to the GW and Bethe-SalpeterGreen's function formalisms. Philos. Trans. R. Soc. A 372, 20130271 (2014).

50. Koslowski, S. et al. Adsorption and electronic properties of pentacene on thin dielectric decoupling layers. Beilstein J. Nanotechnol. 8, 1388-1395 (2017)

51. Repp, J., Meyer, G., Stojković, S. M., Gourdon, A. \& Joachim, C. Molecules on Insulating films: scanning-tunneling microscopy imaging of individual molecular orbitals. Phys. Rev. Lett. 94, 026803 (2005).

52. Grimme, S., Antony, J., Ehrlich, S. \& Krieg, H. A consistent and accurate ab initio parametrization of density functional dispersion correction (DFT-D) for the 94 elements H-Pu. J. Chem. Phys. 132, 154104 (2010).

53. Thodkar, K. et al. Restoring the electrical properties of CVD graphene via physisorption of molecular adsorbates. ACS Appl. Mater. Interfaces 9, 25014-25022 (2017).
54. Zhang, Y. et al. Probing carrier transport and structure-property relationship of highly ordered organic semiconductors at the two-dimensional limit. Phys. Rev. Lett. 116, 016602 (2016).

55. Lin, Z. et al. Controllable growth of large-size crystalline MoS2 and resist-free transfer assisted with a Cu thin film. Scientific Reports 5, 18596 (2015).

56. Maassen, J., Ji, W. \& Guo, H. First principles study of electronic transport through a Cu (111)| graphene junction. Appl. Phys. Lett. 97, 142105 (2010).

57. Ferretti, A. et al. Mixing of electronic states in pentacene adsorption on copper. Phys. Rev. Lett. 99, 046802 (2007)

58. Xu, Z. \& Buehler, M. J. Interface structure and mechanics between graphene and metal substrates: a first-principles study. J. Phys. Condens. Matter 22, 485301 (2010).

59. Toyoda, K. et al. First-principles study of the pentacene/Cu (111) interface: adsorption states and vacuum level shifts. J. Electron Spectrosc. Relat. Phenom. 174, 78-84 (2009).

60. Toyoda, K., Hamada, I., Lee, K., Yanagisawa, S. \& Morikawa, Y. Density functional theoretical study of pentacene/noble metal interfaces with van der Waals corrections: vacuum level shifts and electronic structures. J. Chem. Phys. 132, 134703 (2010).

61. Lagoute, J., Kanisawa, K. \& Fölsch, S. Manipulation and adsorption-site mapping of single pentacene molecules on Cu (111). Phys. Rev. B 70, 245415 (2004).

62. Smerdon, J., Bode, M., Guisinger, N. \& Guest, J. Monolayer and bilayer pentacene on Cu (111). Phys. Rev. B 84, 165436 (2011).

63. Paramonov, P. B., Coropceanu, V. \& Brédas, J.-L. Electronic and vibronic interac tions at weakly bound organic interfaces: The case of pentacene on graphite. Phys. Rev. B 78, 041403 (2008).

64. Vanin, M. et al. Graphene on metals: a van der Waals density functional study. Phys. Rev. B 81, 081408 (2010).

65. Giovannetti, G. et al. Doping graphene with metal contacts. Phys. Rev. Lett. 101, 026803 (2008).

66. Chen, W., Huang, H., Thye, A. \& Wee, S. Molecular orientation transition of organic thin films on graphite: the effect of intermolecular electrostatic and interfacial dispersion forces. Chem. Commun. 4276-4278 (2008).

67. Lee, W. H. et al. Surface-directed molecular assembly of pentacene on monolayer graphene for high-performance organic transistors. J. Am. Chem. Soc. 133 4447-4454 (2011).

68. Bae, S.-H. et al. Unveiling the carrier transport mechanism in epitaxial graphene for forming wafer-scale, single-domain graphene. Proc. Natl Acad. Sci. 114 4082-4086 (2017).

69. Thodkar, K. et al. Comparative study of single and multi domain CVD graphene using large-area Raman mapping and electrical transport characterization. Phys. Status Solidi 10, 807-811 (2016)

70. Nirmalraj, P. et al. Nanoelectrical analysis of single molecules and atomic-scale materials at the solid/liquid interface. Nat. Mater. 13, 947-953 (2014).

71. Schneir, J. \& Hansma, P. K. Scanning tunneling microscopy and lithography of solid surfaces covered with nonpolar liquids. Langmuir 3, 1025-1027 (1987).

72. Schneir, J. et al. Scanning tunneling microscopy and atomic force microscopy of the liquid-solid interface. J. Vac. Sci. Technol. A 6, 283-286 (1988).

73. Nirmalraj, P. et al. Fingerprinting electronic molecular complexes in liquid. Sci. Rep. 6, 19009 (2016).

C Open Access This article is licensed under a Creative Commons cc) Attribution 4.0 International License, which permits use, sharing, adaptation, distribution and reproduction in any medium or format, as long as you give appropriate credit to the original author(s) and the source, provide a link to the Creative Commons license, and indicate if changes were made. The images or other third party material in this article are included in the article's Creative Commons license, unless indicated otherwise in a credit line to the material. If material is not included in the article's Creative Commons license and your intended use is not permitted by statutory regulation or exceeds the permitted use, you will need to obtain permission directly from the copyright holder. To view a copy of this license, visit http://creativecommons. org/licenses/by/4.0/.

(c) The Author(s) 2018 